\title{
Los desafíos de la ciberseguridad en Chile
}

\author{
Daniel Álvarez Valenzuela
}

Editor

Como buena parte de los lectores de la Revista Chilena de Derecho y Tecnología se ha percatado, en los últimos dos años hemos sido testigos privilegiados de cómo el ciberespacio se ha posicionado como una dimensión de la vida cotidiana en torno a la cual se registra uno de los mayores procesos de innovación tecnológica en la historia de la Humanidad. Ya no solo estamos hablamos de internet o de la tecnificación de los procesos productivos, sino que nos referimos también al desarrollo de la computación cuántica, a las consecuencias del uso de herramientas de inteligencia artificial, a los desafíos éticos y legales de la utilización de sistemas letales autónomos, sin dejar de mencionar la robotización, la expansión de la internet de las cosas o la automatización de procesos críticos, todas tecnologías o desarrollos que nos brindan -o que nos brindarán - un cúmulo de facilidades para el desenvolvimiento de la vida moderna, pero que también pueden exponernos a nuevos riesgos y amenazas.

Estos nuevos escenarios nos obligan a reflexionar acerca de las medidas que debemos adoptar para gestionar adecuadamente esos riesgos, tanto a nivel personal y organizacional, como en el diseño y ejecución de políticas públicas que orienten y regulen a los diversos actores involucrados. En este sentido, cabe destacar los procesos realizados en diversos países de América Latina para contar con documentos de política pública que orienten la acción del Estado, del Gobierno, de las empresas y sus organizaciones gremiales, de la sociedad civil y los ciudadanos en materia de ciberseguridad. Así, Colombia, Chile, Paraguay, Costa Rica y México ya han lanzado sus propias políticas o estrategias nacionales de ciberseguridad.

En el caso de Chile, luego de más de tres años de trabajo, se aprobó la Política Nacional de Ciberseguridad, que es el primer instrumento del Estado que tiene por objeto resguardar la seguridad de las personas y de sus derechos en el ciberespacio, estableciendo cinco objetivos estratégicos y un conjunto de medidas que se deben adoptar para contar con un ciberespacio libre, abierto, seguro y resiliente.

Las medidas propuestas por la Política Nacional de Ciberseguridad tienen en especial consideración que Chile ostenta una de las mayores tasas de penetración de internet en América Latina, con más de un $70 \%$ de su población conectada, lo que 
permite que cada vez más y más ciudadanos puedan desenvolverse en este ambiente digital, aprovechando las ventajas de la mensajería instantánea, la amplitud de las redes sociales y la variedad de plataformas de comercio electrónico.

Sin embargo, este incremento en el uso aumenta también nuestra dependencia y vulnerabilidad frente a las redes, que hoy se expresa en la ocurrencia, cada vez más frecuente, de incidentes y ataques informáticos. Esto nos obliga a mejorar los estándares de seguridad informática del país, a encarar eficazmente los ciberdelitos y a garantizar a la población el ejercicio de sus derechos en el ciberespacio.

Una de las mayores dificultades que se experimentó en el proceso de diseño de la Política Nacional de Ciberseguridad es la falta de estudios e información sobre el estado de la seguridad digital, tanto en el sector público como privado, ya sea por el bajo nivel de madurez que tenemos en este ámbito como también por la resistencia de las organizaciones a develar sus debilidades. De ahí la importancia de comenzar a realizar estudios, ya sean de corte doctrinario o informes, que permitan ir dilucidando los efectos que los diversos aspectos de la ciberseguridad pueden ir provocando para las ciencias jurídicas y el derecho, tanto a nivel regional como nacional.

Por ello, en los próximos números de esta revista intentaremos abordar asuntos relativos a la relación entre privacidad y ciberseguridad, las discusiones del derecho internacional público sobre conceptos esenciales para la ciberdefensa, la discusión sobre el uso de estándares y normas técnicas para abordar dimensiones demasiado líquidas para quedar reguladas en normas de carácter legal, conocidas por su rigidez y falta de actualidad, por mencionar solo algunos de las discusiones que esperamos acoger en nuestras páginas.

Finalmente, quisiera aprovechar de informar a nuestra comunidad que la Revista Chilena de Derecho y Tecnología ha sido recientemente aceptada en el prestigioso índice Scopus de Elsevier, que se suma a los índices Emerging Sources Citation Index de Clarivate Analytics, SciELO Chile, Red Iberoamericana de Innovación y Conocimiento Científico (REDIB), Directory of Open Access Journals (DOAJ) y Latindex.

El presente número es resultado del trabajo colaborativo de los ayudantes ad honorem del Centro de Estudios en Derecho Informático, Eduardo Vilches y Máximo Saavedra, del apoyo constante de las autoridades de la Facultad de Derecho y de su Dirección de Investigación, de los profesionales del Sistema de Información y Bibliotecas (SISIB) de la Universidad de Chile y el equipo de Tipográfica, a quienes agradecemos y dedicamos este número. 


\title{
REVISTA CHILENA DE DERECHO Y TECNOLOGÍA
}

La Revista de Chilena de Derecho y Tecnología es una publicación académica semestral del Centro de Estudios en Derecho Informático de la Facultad de Derecho de la Universidad de Chile, que tiene por objeto difundir en la comunidad jurídica los elementos necesarios para analizar y comprender los alcances y efectos que el desarrollo tecnológico y cultural han producido en la sociedad, especialmente su impacto en la ciencia jurídica.

\author{
EDITOR GENERAL \\ Daniel Álvarez Valenzuela \\ (dalvarez@derecho.uchile.cl) \\ SITIO WEB \\ rchdt.uchile.cl \\ CORREO ELECTRÓNICO \\ rchdt@derecho.uchile.cl \\ LICENCIA DE ESTE ARTÍ́CULO \\ Creative Commons Atribución Compartir Igual 4.0 Internacional
}

\begin{abstract}
y
La edición de textos, el diseño editorial

y la conversión a formatos electrónicos de este artículo

estuvieron a cargo de Tipográfica

(www.tipografica.cl).
\end{abstract}

